\title{
Combined Infrared Stereo and Laser Ranging Cloud Measurements from Shuttle Mission STS-85
}

\author{
Redgie S. Lancaster \\ Goddard Earth Science and Technology Center \\ University of Maryland, Baltimore County \\ James D. Spinhirne \\ NASA Goddard Space Flight Center
}

Submitted: November 2001

Journal of Atmospheric and Oceanic Technology

Correspondence: Dr. Redgie Lancaster

NASA Goddard Space Flight Center

Code 912

Greenbelt MD 20771

lancaster@virl.gsfc.nasa.gov 
Authors: Redgie S. Lancaster and James D. Spinhirne,

Title: Combined Infrared Stereo and Laser Ranging Cloud Measurements from Shuttle Mission STS-85

Journal: Journal of Atmospheric and Oceanic Technology

Date: November 2001

\section{Summary}

Multi-angle remote sensing provides a wealth of information for Earth and climate monitoring, such as the ability to measure the height of cloud tops through stereoscopic imaging. Indeed, cloud height is an important driver in the radiative balance of the atmosphere and an accurate global characterization of it is critical for predictive climate and meteorological research. Further, as technology advances so do the options for developing spacecraft instrumentation versatile enough to meet the demands associated with these types of measurements. One such instrument is the Infrared Spectral Imaging Radiometer, which flew as part of mission STS-85 of the space shuttle Columbia in 1997 and was the first Earth-observing radiometer to incorporate an uncooled microbolometer array detector as its image sensor. Specifically, a method for computing cloud-top height from the multi-spectral stereo measurements acquired during this flight has been developed and the results are compared with coincident direct laser ranging measurements from the Shuttle Laser Altimeter. Mission STS-85 was the first space flight to combine laser ranging and thermal IR camera systems for cloud remote sensing. 


\begin{abstract}
Multi-angle remote sensing provides a wealth of information for earth and climate monitoring. And, as technology advances so do the options for developing instrumentation versatile enough to meet the demands associated with these types of measurements. In the current work, the multiangle measurement capability of the Infrared Spectral Imaging Radiometer is demonstrated. This instrument flew as part of mission STS-85 of the space shuttle Columbia in 1997 and was the first earth-observing radiometer to incorporate an uncooled microbolometer array detector as its image sensor. Specifically, a method for computing cloud-top height from the multi-spectral stereo measurements acquired during this flight has been developed and the results demonstrate that a vertical precision of $\pm 0.6 \mathrm{~km}$ was achieved. Further, the accuracy of these measurements is confirmed by comparison with coincident direct laser ranging measurements from the Shuttle Laser Altimeter. Mission STS-85 was the first space flight to combine laser ranging and thermal IR camera systems for cloud remote sensing.
\end{abstract}




\section{Introduction}

Satellite meteorology contributes an incredible wealth of information about the atmosphere of the earth and its environment, primarily through the collection and dissemination of imagery. This imagery is acquired using camera systems placed onboard geosynchronous and polar orbiting spacecraft, and provides operational data about global and regional weather patterns critical to a large portion of society, particularly during times of severe storms. However, the field of satellite meteorology is built upon atmospheric remote sensing and the information acquired using remote sensing techniques is necessarily incomplete. An example of such a shortcoming in the imagery obtained from meteorological satellites is that the cloud vertical dimension is only indirectly inferred. Although this may not be of great practical import in general, it is difficult to deny the importance of the vertical dimension of the atmosphere or its importance for many aspects of predictive climate and meteorological research.

Because remote sensing observations provide only limited information it is generally necessary to couple the data obtained from multiple measurement techniques to provide a more complete picture of the atmosphere. Such is the case with observations designed to remotely measure the height of cloud-tops, where it is generally necessary to rely upon calibrated imagery of a cloud at several wavelengths and an independent knowledge of the atmospheric temperature structure in arriving at a height estimate. Several methods of this sort have been developed such as the IR window technique, the $\mathrm{CO}_{2} / \mathrm{IR}$ window ratio technique, and the $\mathrm{H}_{2} \mathrm{O} / \mathrm{IR}$ window intercept method. A review of these is provided by Nieman et al., [1993]. The approach adopted in the current work departs considerably from these methods, utilizing a purely geometric retrieval of cloud-top height that recovers the vertical information of the imagery through the application of stereoscopy. 
Meteorologists have long recognized the value of using satellite imagery to obtain stereo height measurements of cloud-tops. Indeed, some of the earliest examples of this effort date back to the NASA Nimbus Technology satellite program of the early 1960's. Following a suggestion by Ondrejka and Conover, [1966], imagery collected by the Nimbus II meteorological satellite was used by Kikuchi and Kasai, [1968] to yield estimates of cloud height that displayed a vertical precision of about $2.5 \mathrm{~km}$. Similarly, Whitehead et al., [1969] and Shenk et al., [1975] retrieved estimates of cloud height from several overlapping photographs obtained during the unmanned flight of Apollo 6 in 1968, demonstrating a measurement precision of approximately $\pm 0.5 \mathrm{~km}$.

With the launch of NASA environmental satellites SMS-1 and SMS-2 in 1974 and 1975, however, the focus of stereo remote sensing shifted to the use of imagery obtained from geosynchronous orbit [Bristor and Pichel, 1974]. This imagery is collected using spin-scan radiometers that required approximately 20 minutes to build up an image over their field of view. Hence, to be useful for stereo analysis it is necessary to synchronize the operation of these instruments. Having overcome this operational hurdle, Minzer et al., [1978] demonstrated singlepoint estimates of cloud height that were repeatable to within $\pm 0.5 \mathrm{~km}$. Similar experiments were also performed using radiometers onboard the recently launched NOAA GOES-East and GOESWest satellites in the latter 1970's, resulting in several cases studies from which the utility of stereo imagery was evaluated [Hasler, 1981].

These coordinated experiments and the work of Hasler, [1981] formed the basis of several investigations including those of severe thunderstorms [Fujita, 1982; Fujita and Dodge, 1983; Mack et al., 1983], hurricanes [Mack et al., 1983; Rodgers et al., 1983] and cloud emissivity [Szejwach et al., 1983]. Additionally, multi-satellite observations were extended to include combinations such as the GOES-West and the Japanese GMS-1 geosynchronous satellite [Fujita, 
1982], and the GOES-East and TIROS-N polar orbiting satellites [Hasler et al., 1983]. Through these experiments the importance of stereo imagery as a diagnostic tool for satellite meteorology and cloud remote sensing was powerfully demonstrated. It should be noted, however, that at this time considerable human effort and interpretation was required to retrieve height estimates from stereo observations. And, it was not until the advent of multiprocessor computer systems and the work of Hasler et al., [1991] that this process was largely automated. Today, much more powerful computer systems are available yet with the exception of Wylie et al., [1998] and Mahani et al., [2000], the use of GOES satellites for further stereo imaging experiments has received little attention.

Around the same time that geosynchronous satellite imagery was most widely used for stereo retrievals, Lorenz, [1985] was advocating the use of the Along-Track Scanning Radiometer (ATSR) for the retrieval of cloud-top heights and cloud motion winds from low earth orbit. The ATSR scans in a circular motion with the forward scan meeting the ground track under a nadir angle of 55 degrees, while the backward scan points vertically downward [Prata et al., 1990]. Launched aboard ERS-1 in 1991, this instrument acquires imagery in four channels that include both short wave and thermal infrared. These data have been used by Prata and Turner, [1997] to demonstrate the retrieval of cloud heights with a nominal precision of $\pm 1 \mathrm{~km}$. Fiona et al. [2001] have used similar imagery from ATSR-2, launched in 1995, to provide cloud identification, amount, and altitude at high latitudes.

Lastly, the Multiangle Imaging Spectro-Radiometer (MISR) instrument was launched aboard the Terra spacecraft in 1999 with nine push-broom cameras pointing both fore and aft, at angles as large as 70.5 degrees off of nadir [Diner et al., 1998]. Each camera includes four linear array detectors that acquire data in four spectral channels ranging from the blue to the near-IR. 
According to modeling studies of Horvath and Davies, [2001], the MISR instrument is expected to provide height estimates with a vertical precision of $300-400 \mathrm{~m}$. However, the choice of wavelength channels restricts such measurements to sunlit conditions only.

The current work presents cloud stereo measurements made with the Infrared Spectral Imaging Radiometer (ISIR), flown aboard mission STS-85 of the space shuttle Columbia in 1997. This instrument was the first among earth and climate imaging systems to employ an uncooled microbolometer array detector as its image sensor. And, although it was not designed specifically as a stereo imaging instrument use of this extended array makes such measurements readily available. It is the goal of the current work to examine the utility of the ISIR instrument design and detector technology as the basis of an operational cloud stereo imaging system. This begins with a description of the instrument and the measurements that were made with it from the Space Shuttle. Sample stereo results are presented and the estimated cloud heights are compared to ranging data obtained simultaneously with the Shuttle Laser Altimeter [Bufton, 1989; Garvin et al., 1998]. The paper concludes with a discussion of these results within the context of the requirements of an operational, infrared stereo imaging system.

\section{ISIR Instrument Description}

The Infrared Spectral Imaging Radiometer was developed around an uncooled microbolometer array detector for the purpose of assessing the potential of this technology as a space-borne radiometric imaging sensor. The advantage offered by this technology over other IR detectors is that the microbolometer array provides sensitive thermal infrared imagery without the need for cryogenic, mechanical, or electrical cooling systems. As such there is a large commercial market for these detectors particularly in the production of night-vision cameras for law enforcement and 
military personnel. However, these detectors provide sensitivity in excess of what is required for these commercial applications and which approaches the $120 \mathrm{mK}$ resolution requirement that is typical of infrared radiometers used in satellite meteorology. This high performance combined with the elimination of the need for cooling make microbolometer arrays attractive devices around which to develop infrared payloads tailored to the demands of small satellite missions.

The ISIR instrument was designed and built by NASA Goddard Space Flight Center and Space Instruments Incorporated, under the auspice of the Small Business Innovative Research program. It is a compact spectral pushbroom imager that provides calibrated infrared measurements in three 1micron wide spectral bands centered at wavelengths of 8.55-, 10.2-, and 11.8 microns. A fourth channel is included for measuring broadband radiation covering the spectral region of 7 to $13 \mathrm{um}$. The spatial resolution of the imagery is approximately $250 \mathrm{~m}$ with a swath width of $90 \mathrm{~km}$, when operated from Shuttle orbit. The IFOV of each pixel is $903 \pm 3 \mathrm{urad}$.

A primary objective in the development of the ISIR instrument was to arrive at a compact design that could be easily accommodated within a Shuttle Hitchhiker vessel known as a Get Away Special (GAS) can. Shown in Figure 1 is the resulting instrument design, which includes an optics module, an electronics module, and a calibration assembly. Also included within the instrument is an onboard blackbody source whose temperature is controlled using a stack of thermoelectric coolers. This source is mounted outside of the optical axis and periodically the moveable calibration assembly is positioned such that it projects an unfocused image of the calibration source through the lens assembly and onto the focal plane. When obtaining imagery of the earth this calibration arm remains outside of the optical path.

The optics module is composed of a multi-element lens system, four spectral filters, and the infrared detector package. The lens assembly images directly onto the focal plane with a speed of 
F/0.8 and is optimized for operation in the 8- to 14 um wavelength region. The spectral filters are each one inch in diameter and are composed of several multi-layer interference coatings deposited upon $\mathrm{Ge} / \mathrm{ZnSe}$ substrates. Each is mounted within a filter wheel that positions them directly above the detector focal plane. The electronics module houses 8 printed circuit boards that control the operation of and communication with the camera. These are located in close proximity to the detector to reduce both instrument volume and electrical noise pickup. The ISIR instrument also includes two large-capacity, $8 \mathrm{~mm}$ tape drives capable of storing 14GBytes of data when compressed at an average 2:1 compression ratio. These tape drives are not included in Figure 1.

The uncooled infrared array detector incorporated into the ISIR instrument is an early prototype model manufactured by Lockheed Martin Infrared Systems. It provides a format of 327x246 pixels each $46.25 \mathrm{um}$ in size. The array is assembled into a housing package, which is covered with a thin germanium window and sealed hermetically. This package is then integrated onto a front-end focal plane board that provides the readout electronics. Also included in this imaging module are a video signal processing and controller electronics card, and a power supply card. The imaging module provides a rolling readout of the detector array with 14-bit precision at a rate of 60 frames/second.

\section{Experiment Description}

The Infrared Spectral Imaging Radiometer was included as a Hitchhiker payload experiment onboard the space shuttle Columbia as part of mission STS-85 in August of 1997. During this mission nearly 60 hours of multi-spectral earth imagery was collected over a period of 10 days. These data were obtained in four thermal IR channels with the spectral selection being accomplished through the use of a filter wheel. A complete cycle of the four filters required approximately 6.67 seconds; a period dictated by the requirement for contiguous imagery and made 
necessary by the Shuttle altitude. The ISIR instrument flew concurrently with several other Hitchhiker payloads including the Shuttle Laser Altimeter. The SLA instrument is a surface ranging lidar that additionally provides direct detection measurements of cloud-top height. Mission STS-85 was the first space flight to combine a laser ranging instrument and a thermal IR camera for cloud remote sensing.

The use of an extended array detector in ISIR, coupled with the forward motion of the Space Shuttle, made possible the use of a frame averaging technique known as Time Delay and Integration. Using this technique, the $\mathrm{S} / \mathrm{N}$ ratio of the acquired imagery is improved by integrating successive rows of detectors in a time-delayed sequence, adding the signals from pixel elements originating at the same earth location. This approach requires that the readout of the camera be synchronized with the forward motion of the orbiting platform. Given the design of the ISIR optics and the limitations of the detector electronics the required synchronization was optimal at an orbital altitude of 140 nautical miles. The Shuttle operated at an altitude of approximately 160 nautical miles for most of mission STS-85. During this time imagery was acquired, but with fractional pixel blur. On Day 10 a burn of the main thruster was performed to reduce the altitude to accommodate high-resolution imagery by the ISIR experiment for the last two days of the mission.

The use of TDI necessarily imposes constraints upon the attitude of the orbiting platform, the severity of which depends upon the number of successive rows desired in the Time Delay and Integration average. For ease of computation, it is desirable to restrict the forward motion of the platform to one dimension of the detector array. Doing so simplifies considerably the relative registration of the imagery and allows for precise timing of the detector readout. The rotation of the earth, however, presents a challenge to maintaining this alignment as its contribution to the scene velocity varies with latitude. Thus, to obtain the highest pixel resolution it is necessary to 
continually slew the instrument yaw to counter this effect and maintain the desired alignment. In the measurements of the current work the attitude thrusters of the Shuttle provided the necessary slew adjustments.

During the majority of mission STS-85 the Shuttle orientation was maintained relative to the ground velocity vector with a precision of 0.5 degrees. Upon reducing the orbital altitude to 140 nautical miles, the Shuttle was operated in a special low cross-track motion, or 'Zero-Doppler', steering mode. This operating mode provided a precision of 0.1 degrees relative to the ground velocity vector. At the higher orbital altitude 10 rows were included in the TDI average. When at an altitude of 140 nautical miles the size of the TDI average was increased to include 40 rows. In either case the cross-row drift did not exceed $1 / 8$ pixel during the time required to accomplish the TDI average. The orientation of the detector array relative to the Shuttle was set prior to launch, after receipt of the flight plan.

To provide calibrations of the camera performance an internal blackbody was included within the ISIR instrument. This blackbody was permanently mounted off-axis and a moveable mirror was used to project an image onto the detector when a calibration was required. Additionally, the Shuttle often performed attitude maneuvers in support of the 14 other Hitchhiker experiments included on this mission. Hence, views to deep space were typically acquired just prior to and just after nadir observations of the earth. Together, these observations of cold space and those of the internal blackbody provided two-point calibrations. Measurements of the internal blackbody were performed approximately every 5 minutes. Observations of space were obtained every 30 to 60 minutes.

\section{Methodology}


Stereoscopic retrieval of cloud height necessarily requires that each scene be viewed from two perspectives. This dual-perspective imagery can be acquired either by using two separate instruments to view the same scene simultaneously or a single instrument to view a scene sequentially from two locations. Both techniques present observational challenges. The use of multiple instruments requires that the analysis contend with differences in instrument design, and unless they are flown in formation stereo imagery will be limited to only those times of coincidental pointing. The challenge presented by using a single instrument is that the two perspectives must be acquired before the cloud scene changes appreciably. Fundamental to the design of the ISIR instrument is the concept of viewing the same scene sequentially from multiple locations.

As stated, a filter-wheel design is implemented to provide multi-spectral imagery with an approximate $16.8 \times 10.4$ - degree FOV focused onto the extended infrared array detector at any given instant. Using this approach the imagery appears to be in continual motion with features entering on one side and exiting on the opposing side in response to the forward motion of the Shuttle. Radiometric samples are obtained sequentially in each of the four spectral channels before the scene has sufficient time to pass. Thus, encoded in the imagery of the spectral channels are different perspectives, a comparison of which reveals objects at different altitudes appearing to move relative to one another as a result of parallax. Quantifying this apparent motion provides the necessary mechanism for retrieving the altitude information.

This quantification begins by locating common scene features in the imagery of a pair of spectral channels. To do so, at least two pixels must be used to define a brightness variation in the reference image that is subsequently identified in the search image, with the presence of noise increasing this minimum pixel requirement. In the current work the reference image is divided into a coarse grid of pixel regions, each element of which is registered by searching out the same 
brightness variation in the companion search image. The best match is assumed to be at that location where the value of $\chi^{2}$ is a minimum. Considerable a priori knowledge of the relative location of the chosen window region within the imagery of the two spectral channels exists as both the orbital motion of the spacecraft and the pointing of the instrument are well known. Thus, when performing this relative registration the search is restricted to a small region about the expected position. Additionally, the search is restricted to one dimension of the image as the Shuttle attitude was controlled to limit the amount of cross-track motion that occurred during the collection of data to less than a single pixel.

Once a match is found between the reference and search images, each pixel within the selected window region is assigned the determined value of displacement and the search is performed for the next window region. The optimal choice of window size that should be used in this pattern matching procedure is not known a priori. Broadly, it can be defined as that which maximizes the probability of high correlation, but this depends upon the scale of the image features and the performance of the detector. Previous researchers have addressed this shortcoming by employing telescoping techniques [Hasler et al., 1991], or through experimentation with a variety of window sizes [Fiona et al., 2001]. In the current work, the adopted approach is to repeat the registration for several choices of grid resolutions thereby providing several estimates of displacement for each pixel, from which a weighted average is subsequently calculated. The highest resolution grid that is used consists of $3 \times 3$-pixel regions. The lowest resolution grid is made up of window regions that are $22 \times 22$-pixels in size.

The uncooled infrared array detector used in the ISIR instrument was an early prototype model that exhibited a considerable amount of fixed pattern noise. The onboard blackbody source is used to remove the majority of this noise through frequent calibrations. However, for regions of an 
image showing little variation in brightness, a high probability remains that the registration algorithm will search out the residual noise pattern rather than the desired feature. When this occurs, the registration of a pixel will be erroneous and the search algorithm returns identical estimates of feature displacement regardless of the grid resolution that is used. When not dominated by fixed pattern noise, the search algorithm returns estimates of feature displacement exhibiting a statistical variation for each pixel. Those pixels that do not exhibit at least a 5 percent scatter in registration among the different grid resolutions are discarded and replaced through triangulation.

After the average displacement has been calculated for each pixel, a correction is applied to normalize the resulting values to that of the nadir pixel. Even though each pixel shares a common angular IFOV the nadir pixel represents the smallest footprint and those pixels at the edges of the detector represent the largest. As a result, features sharing a common altitude translate across the image at different rates depending upon their location on the detector with the rate of translation being greater across those pixels representing smaller footprints. Hence, without this correction stereo cloud heights are underestimated for those pixels not at the center of the array. The top panel of Figure 2 illustrates the sinusoidal variation of the footprint across the image and the bottom panel shows the corresponding correction that must be applied to the values of pixel displacement. These results were modeled using the measured value of $903 \pm 3$ urad for the IFOV.

The normalizing correction shown in the lower panel of Figure 2 is repeated four times within an image. This banding is illustrative of the manner in which the spectral channels are combined to provide contiguous stereo results. Four filters were cycled in front of the detector array to provide multi-spectral imagery. And, due to the forward motion of the Shuttle the scene moves roughly $1 / 4$ of the way across the image during the time allotted to a single channel. The imagery obtained in any two consecutive channels thus contains overlapping scene information for approximately $3 / 4$ of 
the image, albeit from slightly different perspectives. The stereo analysis is performed using those spectral channel pairs that have only $1 / 4$ of an image in common, with the common imagery appearing at opposing sides of the detector array. It is in this circumstance that the maximum 7.8degree change in perspective is achieved.

The data displayed in Figure 2 are model results and in practice it is more reliable to apply a correction that has been determined experimentally using imagery of broken, single-altitude cloud layers that are nearby in the orbit. The requisite broken cloud imagery is easily identifiable as the attendant clouds exhibit a largely uniform brightness in the infrared. An example of an experimentally determined correction is shown in Figure 3. Surface imagery can also be used in some cases. However, as ISIR was not optimally designed for surface measurements the preferred approach is one of using cloud scenes. Finally, it should be noted that the imagery from all four spectral channels is required to obtain contiguous stereo results.

\section{Measurements}

The stereo retrieval algorithm described above has been applied to imagery collected with the ISIR instrument during mission STS-85 and the results of three representative cases have been chosen for presentation. These have been selected with the goals of demonstrating 1) the feasibility of the stereo retrieval algorithm, 2) the accuracy of the stereo height retrievals, and 3) the vertical precision with which the height estimates are achieved. The first example employs imagery of a cloud scene that exhibits well-defined features and which readily facilitates qualitative visual confirmation of the stereo results. The second illustrates the accuracy of the stereo results using imagery of an optically opaque cloud system that spans several kilometers in altitude. The third provides an estimate of the vertical precision of the stereo measurements using imagery of a single- 
altitude, broken cumulus cloud layer over a uniform temperature ocean. In the latter two cases the stereo height estimates are compared with the results of direct detection ranging using the Shuttle Laser Altimeter.

The first example of data collected during the ISIR experiment is shown in Figure 4. These data represent an approximate 18 -second segment of more than 60 hours of data, and are a compilation of three individual frames. The forward motion of the shuttle is in the long dimension of the image and the aspect ratio matches that of the camera. The panel on the left contains a false-color image of the radiance as measured through the 10.2 um channel of the instrument. The panel on the right contains the corresponding cloud-top heights that were retrieved using the stereo algorithm described above. This imagery is particularly useful as a first test of the stereo retrieval algorithm as it contains strong cloud features at several different altitudes including a distinct aged contrail. As is seen here, the results of the stereo analysis distinguish between the various cloud layers, revealing the low-level cumulus clouds to be at an altitude around $1.5 \mathrm{~km}$, the mid-level clouds near $5 \mathrm{~km}$, and the contrail reaching as high as $8 \mathrm{~km}$.

When viewed in the image of calibrated radiance the mid-level cloud near $5 \mathrm{~km}$, located in the upper right hand corner of the images of Figure 4, appears to be a possible mixture of semitransparent clouds at different altitudes. Such cloud layers present an obstacle to the accurate retrieval of height using stereo techniques and the utility of this method is often reduced as a consequence. The reason being that the blend of partially transmitting layers makes pattern matching difficult and less well defined. The stereo technique implemented here uses a simple pattern matching algorithm, the ability of which to distinguish partially transmitting clouds is limited. As such, the $5 \mathrm{~km}$ estimate that result for this mixture of clouds is likely an average of 
those above and below this level. More sophisticated algorithms are required to separate the various semi-transparent cloud features.

During mission STS-85 the shuttle Columbia became the first space-based platform to combine laser ranging and thermal IR cloud remote-sensing payloads. The resulting combined data set is thus unique in satellite meteorology, providing an unprecedented opportunity to validate the stereo results through a comparison with direct detection measurements. Additionally, the high precision lidar measurements can be used to quantify the variability inherent to the cloud-top, thereby removing the ambiguity between this variability and the vertical precision of the stereo measurement. Prior to launch, the ISIR instrument was aligned to the Shuttle Laser Altimeter so that a direct comparison of the two measurement techniques could be made. The Shuttle Laser Altimeter was designed to collect surface altimetry and was not optimized for cloud detection or profiling. Still, the data that were collected routinely contain height information on cloud-tops that provide meaningful comparisons with the ISIR stereo results.

To validate the accuracy of the stereo retrieval algorithm against the SLA data an optically thick cloud scene that results in strong lidar returns, and which spans a wide range of altitudes is optimal. A quantitative comparison between the ISIR stereo and SLA direct detection results can be achieved by restricting the retrieved stereo cloud heights to only those that share a common field of view with the SLA instrument. Such a comparison is shown in Figure 5 where the solid line represents the results of the stereo retrieval and the diamonds represent the measurements of the SLA instrument. As expected the stereo results show a much higher degree of scatter than do the direct detection lidar measurements. However, the two instruments are clearly measuring the same cloud region and obtaining results for cloud-top altitude that agrees favorably. A notable exception to this conclusion is seen in the data indexed near $25 \mathrm{~s}$, where the cloud is shown by the lidar 
measurements to be multi-layered with surfaces at 4 and $6 \mathrm{~km}$. The scale of this horizontal structure is too fine to be retained by the stereo algorithm.

The stereo cloud height estimates of Figure 5 are restricted to only those pixels that coincide with the ground track of the Shuttle Laser Altimeter. It should be noted, however, that while the footprint of the SLA instrument is contained within a single pixel of the infrared image, an extended region of pixels is required to arrive at a stereo height estimate. In the current work, image features located up to 11 pixels from the pixel to which the result is assigned influence each height estimate. This fact should be recognized when interpreting the results of the stereo height calculations on the scale of a single pixel. Additionally, pixels located within those regions displaying insufficient structure to accommodate the pattern recognition algorithm are assigned height estimates by triangulating nearby results. Thusly, uniform regions of an image can often be neglected and assigned the height of a nearby cloud layer. Such is the case in Figure 6 where the segment of ISIR imagery displays scattered cumulus clouds over a largely uniform temperature ocean.

The results of Figure 6 are used to provide an estimate of the vertical resolution that is achieved with the ISIR stereo measurements. Here the solid curve represents the stereo measurements and the diamonds represent the direct-detection measurements. The direct-detection measurements reveal the average height of the cloud-tops to be at $1753 \pm 179 \mathrm{~m}$. The uncertainty in this value is primarily the result of variations in cloud-top altitude and is not representative of the precision of the direct-detection measurement. The stereo measurements reveal the cumulus clouds to be located at an average altitude of $1826 \pm 620 \mathrm{~m}$. This standard deviation is a measure of the vertical precision achieved by the stereo observations as the variability of the cloud-tops, revealed through the SLA instrument, is small by comparison. Although this precision estimate tends to vary 
somewhat according to the chosen imagery, $\pm 620 \mathrm{~m}$ is representative of the vertical height resolution achieved for a single pixel of the ISIR imagery.

The use of laser profiling to define cloud heights is well know for airborne and ground-based applications. These cloud lidar systems are designed to record an entire profile of the return signal, up to the signal attenuation limit (Spinhirne et al., 1983). As such they provide measurements of both the upper and lower cloud boundaries and a profile of the interior of those clouds that present an optical depth less than unity. For optically thick clouds a region of penetration is sampled and typically the strongest signal is returned from several hundred meters within the cloud, when viewing in the nadir. The SLA data system was not designed to accomplish this measurement but rather to trigger off set signal levels, recording a small portion of the signal immediately prior to and just after the trigger threshold events. And, due to the design of SLA and various noise considerations the trigger threshold was set high relative to signals from cloud tops. Consequently, less dense clouds such as thin cirrus were generally not detected. For those cloud cases where a trigger was obtained, the threshold detection could be as much as kilometers below the higher thin layers of the cloud top. For the more uniform status clouds of Figure 5, and those of Figure 6 that show a consistent detection of the layer, aircraft lidar experience would indicate a true cloud top within a few hundred meters of the detected threshold altitude.

\section{Discussion}

Multiangle remote sensing provides several opportunities for advancing our understanding of geophysical and biophysical parameters [Diner et al., 1999]. Indeed, recent instruments placed in low earth orbit such as ATSR and MISR have been developed specifically for this purpose. Additionally, the network of geosynchronous weather satellites can contribute limited multi-angle 
observations, albeit with considerable effort as they currently are not designed to operate in a manner that easily accommodates the requisite synchronization. As the scientific issues that require multiangle observations are both rich and diverse, additional instrumentation will undoubtedly be required in the future. It is the designs of these instruments that can benefit from the continued advancement of technology offering the potential of greater versatility contained within a design that is more compact and robust.

The Infrared Spectral Imaging Radiometer was the first earth remote-sensing instrument to incorporate an uncooled microbolometer array detector as its image sensor. And, its relatively simple camera design is conducive to providing reliable, operational multiangle imagery. Hence, the potential is great that the ISIR instrument or a minor variant thereof, can provide a powerful stereo remote-sensing tool for satellite meteorology. The current work illustrates this multiangle capability through the stereo inversion of cloud-top height. In the discussion that follows three aspects of the ISIR operation and performance are addressed. These are the achieved vertical height resolution, the optimization of the stereo retrieval algorithm, and the possibility of measuring winds simultaneously with cloud heights.

The cloud height measurements of the current work have been shown experimentally to be precise to within approximately \pm 620 meters. This estimate of vertical resolution was achieved by comparing stereo height measurements with those obtained simultaneously through direct detection, using the Shuttle Laser Altimeter. Such an approach is arguably the optimal one for verifying cloud height and has been advocated by past investigators such as Lorenz, [1985] and Hasler et al., [1991]. As mission STS-85 was the first to overcome the challenges of including both the laser ranging and stereo imaging systems on the same space platform, previous investigators were left to rely upon less reliable characterizations of their achieved measurement precision. Shown in Table 1 
is a summary of all empirical estimates of vertical resolution associated with these previous measurements as well as the current work, and the methods that were used.

As is evident by the dearth of case data in Table 1, obtaining an empirical verification of vertical resolution is an elusive task. The Apollo 6 measurements of Whitehead et al., [1969] and Shenk et al, [1975] were estimated to achieve a vertical resolution of about $\pm 0.5 \mathrm{~km}$ by independently assessing the extent of the shadows cast by the clouds themselves. The investigations of Minzer et al., [1978] and Hasler, [1981] sought out stereo imagery of terrestrial features such as mountains and lakes of known elevation for comparison. Using these features and those of select coastlines they estimated the vertical precision to be as high as $\pm 0.15 \mathrm{~km}$, but more typically $\pm 0.3 \mathrm{~km}$. These features are, however, both extended and well defined and as such their suitability for characterizing the resolution of a cloud measurement is less than ideal. Recognizing this, Hasler, [1981] estimates the achieved vertical resolution to be approximately $\pm 0.5 \mathrm{~km}$ for a given measurement of cloud height. Prata and Turner, [1997] also used terrestrial features to estimate the vertical resolution of the ATSR instrument at approximately $\pm 1 \mathrm{~km}$. The vertical resolution achieved in the current work is approximately $\pm 0.6 \mathrm{~km}$, not considerably less than that of previous investigations and exceeding that of ATSR.

This vertical resolution represents that of a single pixel of the ISIR image. The average cloud height, however, can be identified with considerably more precision by averaging the single pixel results. This is illustrated in Figure 7, wherein the improvement in the vertical height resolution that accompanies pixel averaging is seen. The imagery used for this example is the broken cumulus layer of the previous figure. As stated, the Shuttle Laser Altimeter results show the variability of this cloud-top layer to be approximately $\pm 180 \mathrm{~m}$. The asymptotic precision of the stereo 
measurements in Figure 7 is also approximately this value, requiring a bin size of about 150x150 pixels. This represents a $38 \times 38 \mathrm{~km}$ area of the ISIR measurements.

Vertical resolution tends to be a compromise between the optical design, the detector performance, and the stereo retrieval algorithm. The optical design of ISIR provides a maximum parallax angle of approximately 7.8 degrees and a spatial resolution of about $0.25 \mathrm{~km}$ when in Shuttle orbit. Using these values it is straightforward to calculate that a single pixel of parallax represents roughly a $1.8 \mathrm{~km}$ change in altitude. This approximate agreement with the experimentally determined vertical resolution lends support to the credibility of the stereo inversion algorithm, and in particular, the image registration technique. The primary concern in performing the image registration is that of the optimal selection of the image grid that is used. As stated, the current work divides the search image into a grid of pixel regions, each of which is subsequently registered to a comparison image. And, several factors necessarily influence the optimal choice of grid coarseness including the performance of the detector and the typical scale of the image features. Choosing a grid that is too coarse will reduce the vertical resolution of the stereo results, as neighboring cloud features will not be sufficiently resolved. Choosing a grid that is too fine will also reduce the vertical resolution since the detector noise will impact the results disproportionately.

The optimal choice of grid resolution can be gleaned in the current work by performing a correlation analysis of the stereo height returns for different selections of grid resolution and the image brightness temperatures. Clouds tend to be in near thermal equilibrium with the surrounding atmosphere and the blackbody radiation that is emitted provides a mechanism for measuring the corresponding temperature. Indeed, a common method of estimating cloud height from infrared imagery consists of comparing the measured brightness temperature with an atmospheric profile obtained from a nearby temperature sounding [Nieman, 1993]. This approach to evaluating the 
optimal grid size does have shortcomings as a cloud is not a perfect blackbody and a thin cloud will exhibit an elevated brightness temperature as it transmits much of the radiation from the warm surface below. Thus, the correlation will not be exact but should nonetheless give an indication of the optimal choice of grid size for use in the registration algorithm. This lack of perfect correlation is in itself an argument for using stereoscopic inversion methods to determined cloud height.

Shown in Figure 8 are the results of this correlation study when applied to the imagery of the contrail scene shown in Figure 4. The correlation between cloud height and brightness temperature appears to maximize at around 70-100 pixels, with a 100-pixel region representing a 10x10-pixel grid. The peak of the curve of Figure 8 is not sharp, however, and it is apparent that a host of grid choices ranging from 8x8-pixels to 16x16-pixels provide similar amounts of correlation. The current work obtains stereo height estimates using selections of grid resolution ranging from $3 \times 3-$ pixels to 22x22-pixels. And, the correlation results of Figure 8 are used as a weight function when averaging the estimates from the different grid resolutions. That the correlation is not optimal for a $3 \times 3$-pixel grid resolution is, in part, a reflection of the detector noise limitations, as it is not unusual for the stereo results of as many as $1 / 4$ of the pixels to be discarded and replaced with values obtained through triangulation. Current production model detectors offer a greatly reduced fixed pattern noise and factor of two improvements in radiometric performance.

A primary reason to measure cloud height is to enable the assignment of wind speed when observing cloud motions apparent in a time series of satellite images. Winds gleaned in this manner are known as cloud motion winds and it is the independent measure of cloud height that provides the needed calibration for this measurement. Lorenz, [1981] discusses two approaches to remotely sensing cloud motion winds. The first is to couple multiple cameras that can provide two pairs of stereo imagery. One stereo pair provides an estimate of cloud height that is used to adjust the 
perspective of the second pair for the effects of parallax, leaving only real cloud motion from which to estimate wind speed. This option is implemented in the MISR instrument [Horvath and Davies, 2001]. The second approach is to couple a laser ranging system with a single stereo camera. The laser ranging system provides the requisite independent measure of cloud height that is used together with the stereo imagery to glean estimates of cloud motion that occurred as a result of wind.

The current work presents imagery from a single stereo camera system that is coupled to a laser

ranging system. As such, the second of the above methods is the logical choice for retrieving cloud motion winds from the infrared imagery collected during mission STS-85. The ISIR instrument, however, was not ideally configured for this measurement as less than 6.7 seconds elapsed between the acquisition of the images that make up a stereo pair. With a spatial resolution of approximately $0.25 \mathrm{~km}$, a single pixel of motion represents a wind speed in excess of $37 \mathrm{~m} / \mathrm{s}$. As such, the stereo retrievals that result from the ISIR design are seen to be largely insensitive to typical cloud motions. This lack of sensitivity to cloud motion makes the height measurements of the Shuttle Laser Altimeter particular useful in calibrating the stereo height retrievals. However, the combined stereo and laser ranging measurements are not particularly useful for retrieving wind estimates.

\section{Conclusions}

The ISIR instrument is unique among thermal imaging systems developed for use in satellite meteorology, being the only one thus far to incorporate an uncooled microbolometer array detector as its image sensor. And, because the development of this detector technology is relatively recent considerable uncertainty remains regarding its potential for satisfying the demands of infrared earth 
and climate monitoring systems. However, it is through pilot experiments such as the one reported here that this potential can be assessed. The focus of the current work is upon the possibility of using this detector technology in the development of an operational stereo cloud remote sensing instrument. Toward that end, a stereo algorithm was developed to retrieve estimates of cloud-top height from the multi-spectral imagery collected with this sensor during the STS-85 mission of the space shuttle Columbia. Cloud-top heights were measured with a resolution of approximately \pm 620 $\mathrm{m}$ in good agreement with that anticipated from the optical design. A comparison with direct detection measurements of the Shuttle Laser Altimeter confirms the accuracy of the stereo retrievals.

The ISIR program was designed to generally address the potential of the microbolometer detector array as a space sensor and not specifically to develop a cloud stereo imaging system, even though the current work examines the possibility of using this device for this specific purpose. When placed onboard a spacecraft in low earth orbit rather than on the Shuttle, the vertical height resolution offered by the ISIR design would be reduced by about a factor of two. Hence, to retain the vertical precision either the spatial resolution would need to be increased at the expense of the FOV or a larger format than the $327 \times 246$ pixel array would need to be implemented. Uncooled microbolometer array technology has advanced considerably since the ISIR instrument was built and flown. Today, microbolometer detector arrays are readily available in formats of $640 \times 480$ pixels. Further, only modest technical challenges need to be overcome to produce arrays with formats of $1024 \times 1024$ or larger. The primary hurdle to developing this format is the emergence of a commercial market that demands such a product.

In developing an operational stereo imaging system based upon the ISIR design, a useful modification would be to eliminate the narrow band spectral filters. If the objective of the 
measurement is solely stereo retrieval then there is little reason not to utilize the full spectral response of the detector to maximize the $\mathrm{S} / \mathrm{N}$ of the imagery. In this way the instrument can be made very small, with sizes comparable to that of head-mounted, military night-vision cameras. An instrument of this size could easily be accommodated by a host of satellite platforms. This smaller size also makes possible the use of multiple cameras, each pointing in different directions, instead of the stereo imaging being performed using only a single detector as with ISIR. Similarly, compact stereo instruments could be flown in formation on a constellation of small satellites. Using this approach, it would be possible to measure atmospheric winds as well by observing the amount of cloud motion that takes place in the intervening time between the passage of the two stereo cameras.

Lastly, these measurements provide a good pilot experiment for future satellite meteorology missions that combine infrared stereo imagery and direct-detection lidar. Combined measurements of this sort would, however, benefit from a lidar system that is able to provide a cloud profile rather than merely range altitude. Such a system would provide information about the thickness of a cloud system and the presence of multiple cloud layers; characteristics important to accurately model cloud microphysical properties. The first operational space borne lidar missions are planned for the next few years (Spinhirne and Palm, 1996), but current constraints limit sampling to the nadir for the foreseeable future. Coupling an infrared stereo imaging instrument such as ISIR with these planned missions would provide a unique opportunity to extend the spatial coverage beyond that sampled directly by the lidar and to improve the accuracy and understanding of the passive observations. 


\section{REFERENCES}

Bufton J.L., 1989: Laser altimetry measurements from aircraft and spacecraft. Proc. of IEEE, 77, 463-477.

Bristor, C.L., and W. Pichel, 1974: 3-D cloud viewing using overlapping pictures from two geostationary satellites. Bull. A. Meteorol. Soc., 55, 1353-1355.

Cawkwell, F.G.L, J.L. Bamber, and J-P. Muller, 2001: Determination of cloud top amount and altitude at high latitudes. Geophys. Res. Lett., 28, 1675-1678.

Diner, J.D., J.C. Beckert, T.H. Reilly, C.J. Bruegge, J.E. Conel, R.A. Kahn, J.V. Martonchik, T.P. Ackerman, R. Davies, S.A.W. Gerstl, H.R. Gordon, J-P. Muller, R.B. Myneni, P.J. Sellers, B. Pinty, and M.M Verstraete, 1998: Multi-angle imaging spectroradiometer (MISR) instrument Description and experiment overview. IEEE Trans. Geosci Remote Sens., 36, 1072-1085.

Diner, J.D., G.P. Asner, R. Davies, Y Knyazikhin, J-P. Mueller, A.W. Nolin, B. Pinty, C.B. Schaaf, and J. Stroeve, 1999: New directions in earth observing: scientific applications of multiangle remote sensing, Bull. Am. Meteorol. Soc., 80, 2209-2228.

Fujita, T.T., 1982: Principle of stereoscopic height computations and their applications to stratospheric cirrus over severe thunderstorms. J. Meteorol. Soc. Japan, 60, 355-368.

Fujita, T.T. and J.C. Dodge, 1983: Applications of stereoscopic height computations from dual geosynchronous satellite data/joint NASA-Japan stereo project. Adv. Space Res., 2, 153-160.

Garvin, J., J. Bufton, J. Blair, D. Harding, S. Luthcke, J. Frawley, and D.D. Rowlands, 1998: Observations of the Earth's topography from the Shuttle Laser Altimeter (SLA): laser-pulse echorecovery measurements of terrestrial surfaces. Phys. Chem. Earth, 23, 1053-1068.

Hasler, A.F, 1981: Stereographic observations from geosynchronous satellites: An important new tool for atmospheric sciences. Bull. Am. Meteorol. Soc., 62, 194-212. 
Hasler, A.F., R. Mack, and A. Negri, 1983: Stereoscopic observations from meteorological satellites. Adv. Space Res., 2, 105-113.

Hasler, A.F., J. Strong, R.H. Woodward and H. Pierce, 1991: Automatic analysis of stereoscopic satellite image pairs for determination of cloud-top height and structure. J. Appl. Meteor., 30, 257-281.

Horvath, A. and R. Davies, 1968: Feasibility and error analysis of cloud motion wind extraction from near-simultaneous multiangle MISR measurements. J. Atmos. Ocean Tech., 18, 591-608. Kikuchi, K. and T. Kasai, 1968: Stereoscopic analysis of photographs taken by NIMBUS II APT system. J. Meteorol. Soc. Japan, 46, 60-67.

Lorenz, D., 1985: On the feasibility of cloud stereoscopy and wind determinations with the AlongTrack Scanning Radiometer. Int. J. Remote Sensing, 6, 1445-1461.

Mack, R., A.F. Hasler, and E.B. Rodgers, 1983: Stereoscopic observations of hurricanes and tornadic thunderstorms from geosynchronous satellites. Adv. Space Res., 2, 143-151.

Mahani, S.E., X. Gao, S. Sorooshian and B. Imam, 2000: Estimating cloud top height and spatial displacement from scan-synchronous GOES images using simplified IR-based stereoscopic analysis. J. Geophys. Res., 105, 15,597-15,608.

Minzner, R.A., W.E. Shenk, R.D. Teagle, and J. Steranka, 1978: Stereographic cloud heights from imagery of SMS/GOES satellites, Geophys. Res. Lett., 5, 21-24.

Nieman, S.J., J. Schmetz, and W.P. Menzel, 1993: A comparison of several techniques to assign heights to cloud tracers. J. Appl. Meteor. 32, 1559-1568.

Ondrejka, R.J. and J.H. Conover, 1966: Note on the stereo interpretation of Nimbus II APT photography. Mon. Wea. Rev., 94, 611-614.

Prata, A.J., R.C. Cechet, I.J. Barton, and D.T. Llewellyn-Jones, 1990: The along-track scanning 
radiometer for ERS-1 - scan geometry and data simulation. IEEE Trans. Geosci. Remote Sens., 28, 1-13.

Prata, A.J. and P.J. Turner, 1997: Cloud-top height determination using ATSR data. Remote Sens. Environ., 59, 1-13.

Rodgers, E.B., R. Mack, and A.F. Hasler, 1983: A satellite stereoscopic technique to estimate tropical cyclone intensity. Mon. Wea. Rev., 111, 1599-1610.

Shenk, W.E., R.J. Holub, and R.A. Neff, 1975: Stereographic cloud analyses from Apollo 6 photographs over a cold front. Bull. Am. Meteorol. Soc., 56, 4-16.

Spinhirne, J.D., M.Z. Hansen, and L.O. Caudill, 1982: Cloud top remote-sensing by airborne Lidar. Applied Opt., 21, 1564-1571.

Spinhirne, J.D. and S.P. Palm, 1996: Space based atmospheric measurements by GLAS, in Advances in Atmospheric Remote Sensing with Lidar, A. Ansmann Ed., Springer, Berlin, 213217.

Szejwach, G., T.N. Sletten, and A.F. Hasler, 1983: The use of stereoscopic satellite observation in the determination of the emissivity of cirrus. Adv. Space Res., 2, 161-164.

Whitehead, V.S., I.D. Browne, and J.G. Garcia, 1969: Cloud height contouring from Apollo 6 photography. Bull. Am. Meteorol. Soc., 50, 522-528.

Wylie, D.P, D. Santek and D.O'C. Starr, 1998: Cloud-top heights from GOES-8 and GOES-9 stereoscopic imagery. J. Appl. Meteor. 37, 405-413. 


\section{TABLE CAPTIONS}

Table 1. An empirical determination of the achieved vertical resolution requires an independent measure of cloud height to which the stereo estimates can be compared. Shown here are the published estimates of previous investigators and the methods that were used to arrive at these estimates. 


\section{FIGURE CAPTIONS}

Figure 1. The Infrared Spectral Imaging Radiometer was included among the complement of Hitchhiker instruments aboard mission STS-85. Operating from the space shuttle Columbia, the ISIR instrument was the first space-borne cloud radiometer built around an uncooled microbolometer array detector.

Figure 2. Each pixel of the ISIR instrument shares a common angular IFOV and the corresponding footprint follows a cosine variation (shown in the top panel) about the nadir pixel. Hence, a correction factor (shown in the bottom panel) must necessarily be applied to the resulting cloud height estimates.

Figure 3. The correction factor that must be applied to the cloud height estimates can be gleaned experimentally by performing the stereoscopic height retrieval using imagery of single-layered, broken clouds nearby in the orbit. Such cloud scenes are readily recognizable in the ISIR infrared imagery.

Figure 4. The panel on the left shows a sample of the imagery obtained using the ISIR instrument during mission STS-85. These data have been calibrated into units of brightness temperature, as measured through the 10.2 um channel of the instrument. The panel on the right shows the corresponding estimates of cloud height obtained stereoscopically. Here, a multi-layered cloud system is seen that includes an aged contrail at an altitude near $8 \mathrm{~km}$. 
Figure 5. Prior to launch the ISIR instrument was aligned with its FOV centered on the groundtrack of the Shuttle Laser Altimeter to facilitate a comparison of the measurements provided by the two instruments. Shown here is a comparison of cloud height estimates obtained stereoscopically and through direct detection for an optically thick cloud scene that reaches as high as $6 \mathrm{~km}$ in altitude.

Figure 6. A scene of broken cumulus clouds is used to estimate the vertical resolution of the stereoscopic cloud height measurements by comparing them with direct detection measurements obtained from the Shuttle Laser Altimeter. Inversion of the ISIR stereo imagery results in an average cloud height of $1826 \pm 620 \mathrm{~m}$ whereas the direct detection measurements of the Shuttle Laser Altimeter reveal an average height of $1753 \pm 179 \mathrm{~m}$.

Figure 7. The average height of a cloud layer can be determined more precisely than that of a single pixel through binning the stereo results. This is demonstrated here by binning the imagery of the single cumulus layer seen in the previous figure. The asymptotic precision of the stereo results is seen to be approximately $\pm 180 \mathrm{~m}$ for this cloud layer, in agreement with the cloud-top variability measurements of the Shuttle Laser Altimeter.

Figure 8. The correlation between cloud height and brightness temperature provides a means to determine the optimal grid size for use in the registration of the ISIR imagery. Using the imagery of Figure 4 it is seen that an approximate 10x10 -pixel grid provides the best correlation between 
the brightness temperature and stereoscopically derived cloud height. These results are used to weight the average of stereo height estimates obtained using different grid resolutions. 
Table 1. An empirical determination of the achieved vertical resolution requires an independent measure of cloud height to which the stereo estimates can be compared. Shown here are the published estimates of previous investigators and the methods that were used to arrive at these estimates.

\begin{tabular}{|l|l|l|l|}
\hline Stereo Measurement & Platform & $\begin{array}{l}\text { Vertical } \\
\text { Resolution }\end{array}$ & Verification \\
\hline $\begin{array}{l}\text { Whitehead et al., [1969]; } \\
\text { Shenk et al., [1975] }\end{array}$ & Apollo 6 & $\pm 0.5 \mathrm{~km}$ & Cloud Shadows \\
\hline Minzer et al., [1978] & $\begin{array}{l}\text { GEO, separated by } \\
32^{\circ} \text { longitude }\end{array}$ & Approx. $\pm 0.3 \mathrm{~km}$ & $\begin{array}{l}16 \text { mountain } \\
\text { features, 1 lake }\end{array}$ \\
\hline Hasler, [1981] & $\begin{array}{l}\text { GEO, separated by } \\
60^{\circ} \text { longitude. }\end{array}$ & Approx. $\pm 0.5 \mathrm{~km}$ & $\begin{array}{l}\text { Coastlines, } \\
\text { Mountain lakes }\end{array}$ \\
\hline Prata and Turner, [1997] & Low Earth Orbit & $\pm 1 \mathrm{~km}$ & $\begin{array}{l}\text { Mountain } \\
\text { topography }\end{array}$ \\
\hline Current Work & Space Shuttle & $\pm 0.6 \mathrm{~km}$ & $\begin{array}{l}\text { Simultaneous Cloud } \\
\text { laser ranging }\end{array}$ \\
\hline
\end{tabular}




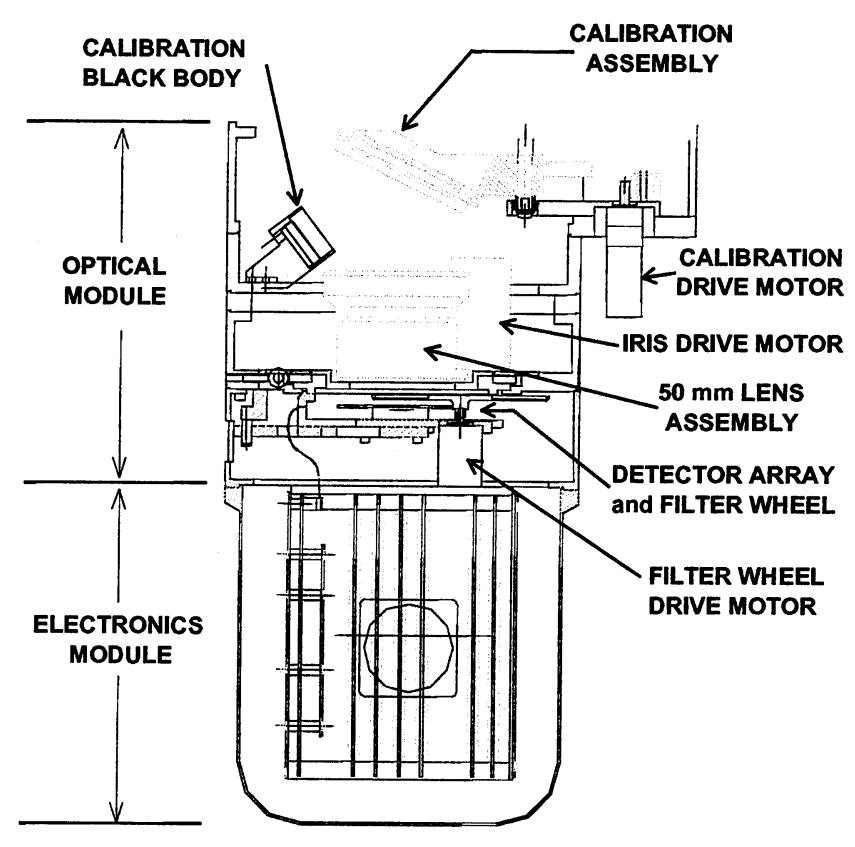

Figure 1. The Infrared Spectral Imaging Radiometer was included among the complement of Hitchhiker instruments aboard mission STS-85. Operating from the space shuttle Columbia, the ISIR instrument was the first space-borne cloud radiometer built around an uncooled microbolometer array detector. 

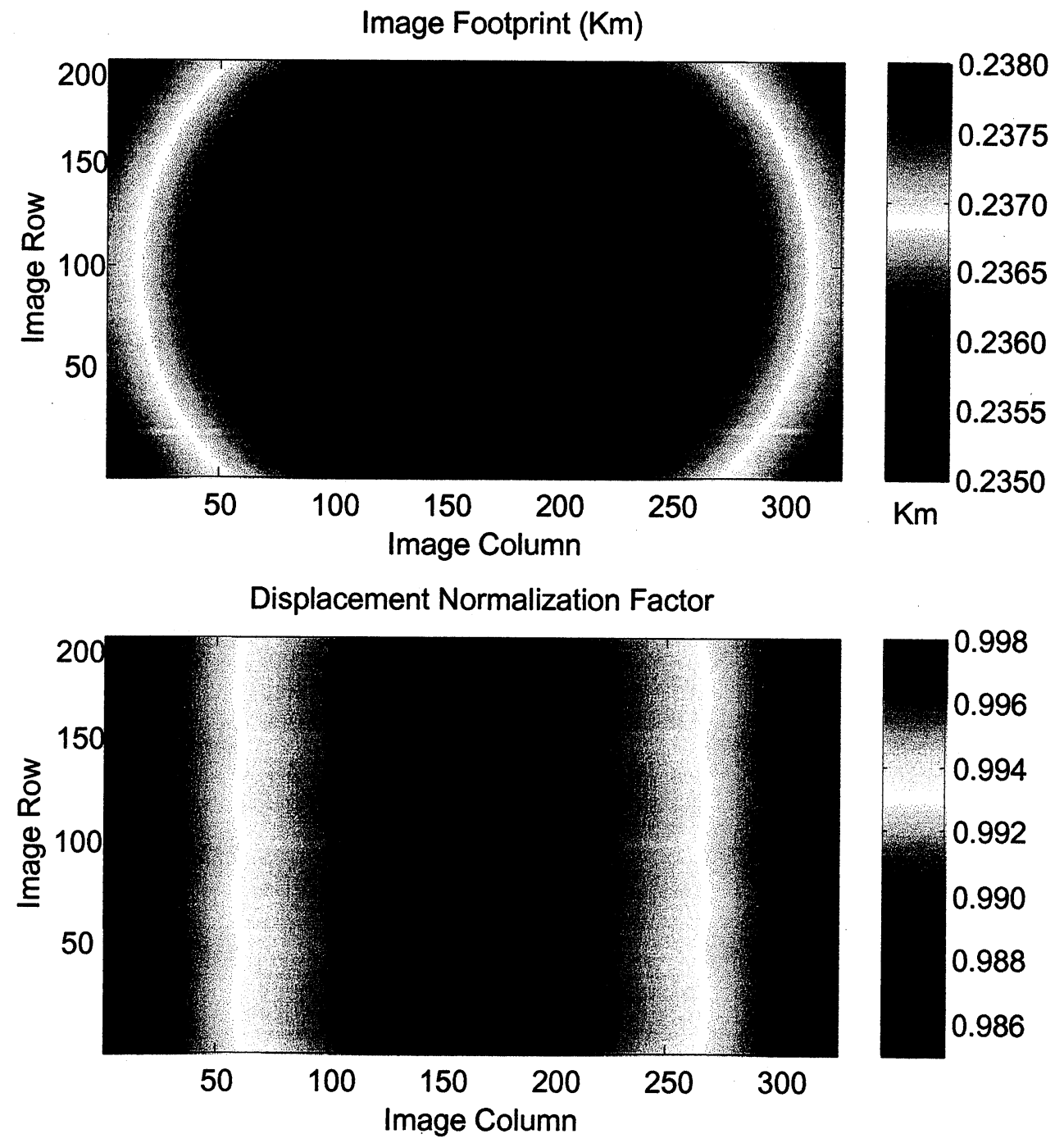

Figure 2. Each pixel of the ISIR instrument shares a common angular IFOV and the corresponding footprint follows a cosine variation (shown in the top panel) about the nadir pixel. Hence, a correction factor (shown in the bottom panel) must necessarily be applied to the resulting cloud height estimates. 


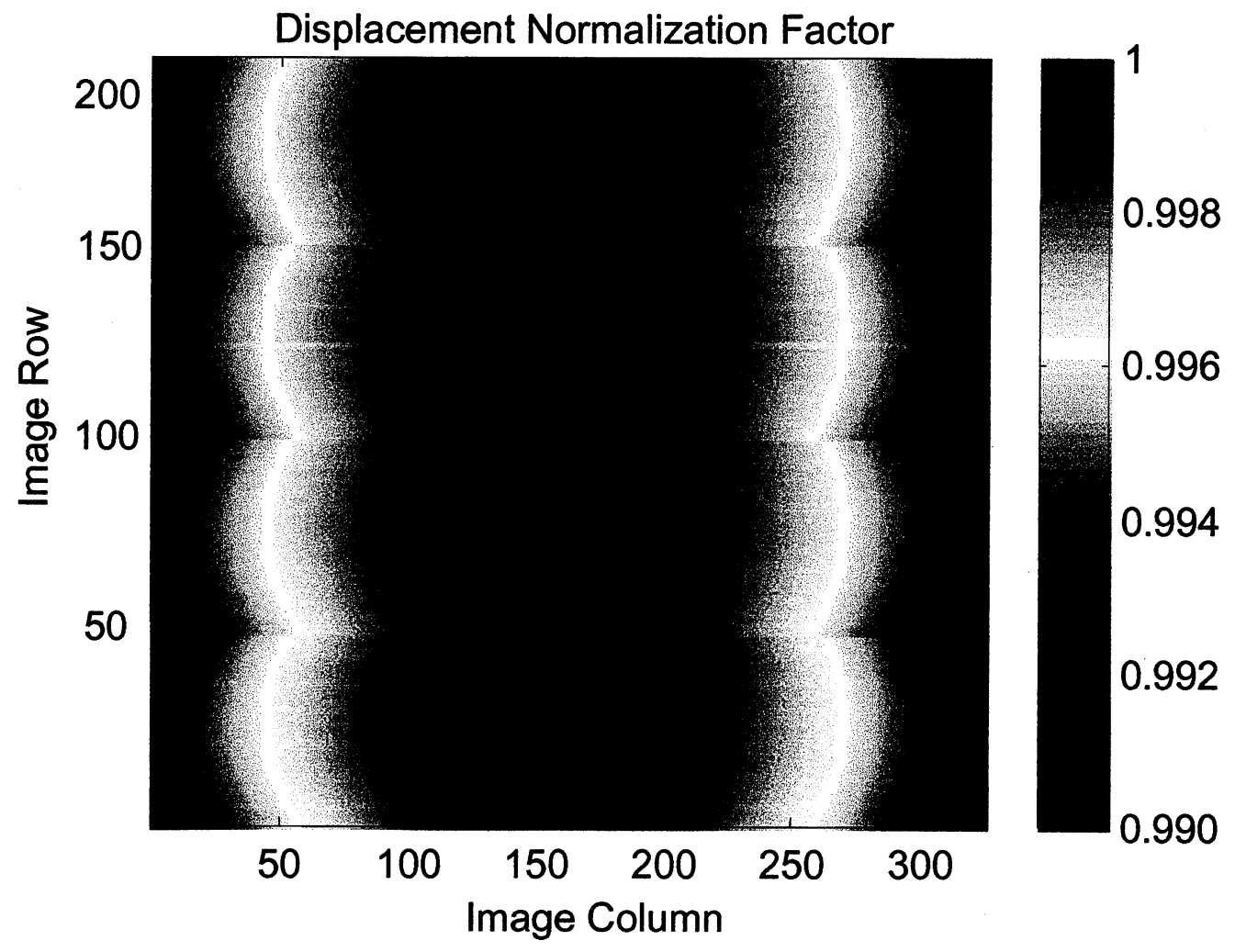

Figure 3. The correction factor that must be applied to the cloud height estimates can be gleaned experimentally by performing the stereoscopic height retrieval using imagery of single-layered, broken clouds nearby in the orbit. Such cloud scenes are readily recognizable in the ISIR infrared imagery. 

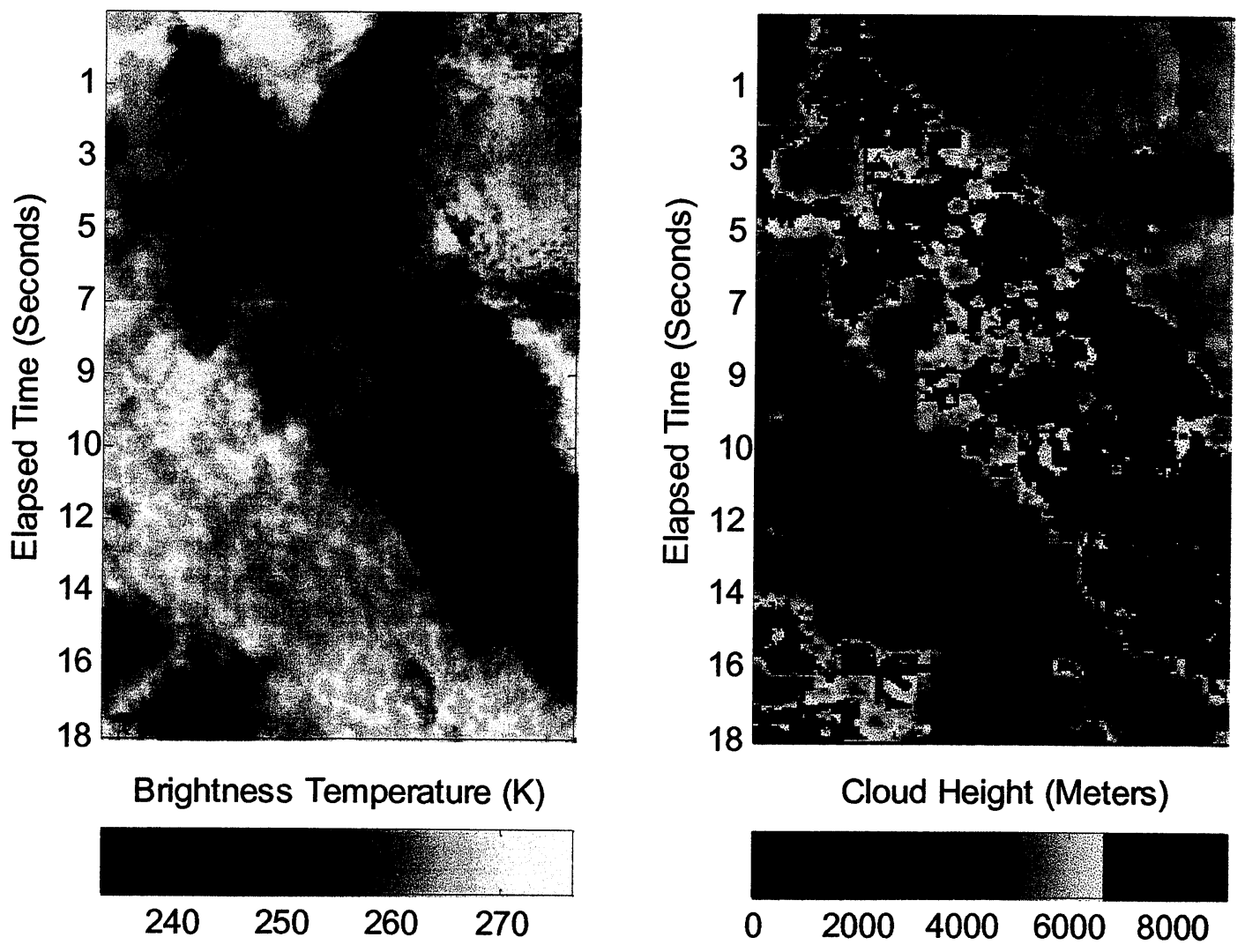

Figure 4. The panel on the left shows a sample of the imagery obtained using the ISIR instrument during mission STS-85. These data have been calibrated into units of brightness temperature, as measured through the 10.2 um channel of the instrument. The panel on the right shows the corresponding estimates of cloud height obtained stereoscopically. Here, a multi-layered cloud system is seen that includes an aged contrail at an altitude near $8 \mathrm{~km}$. 

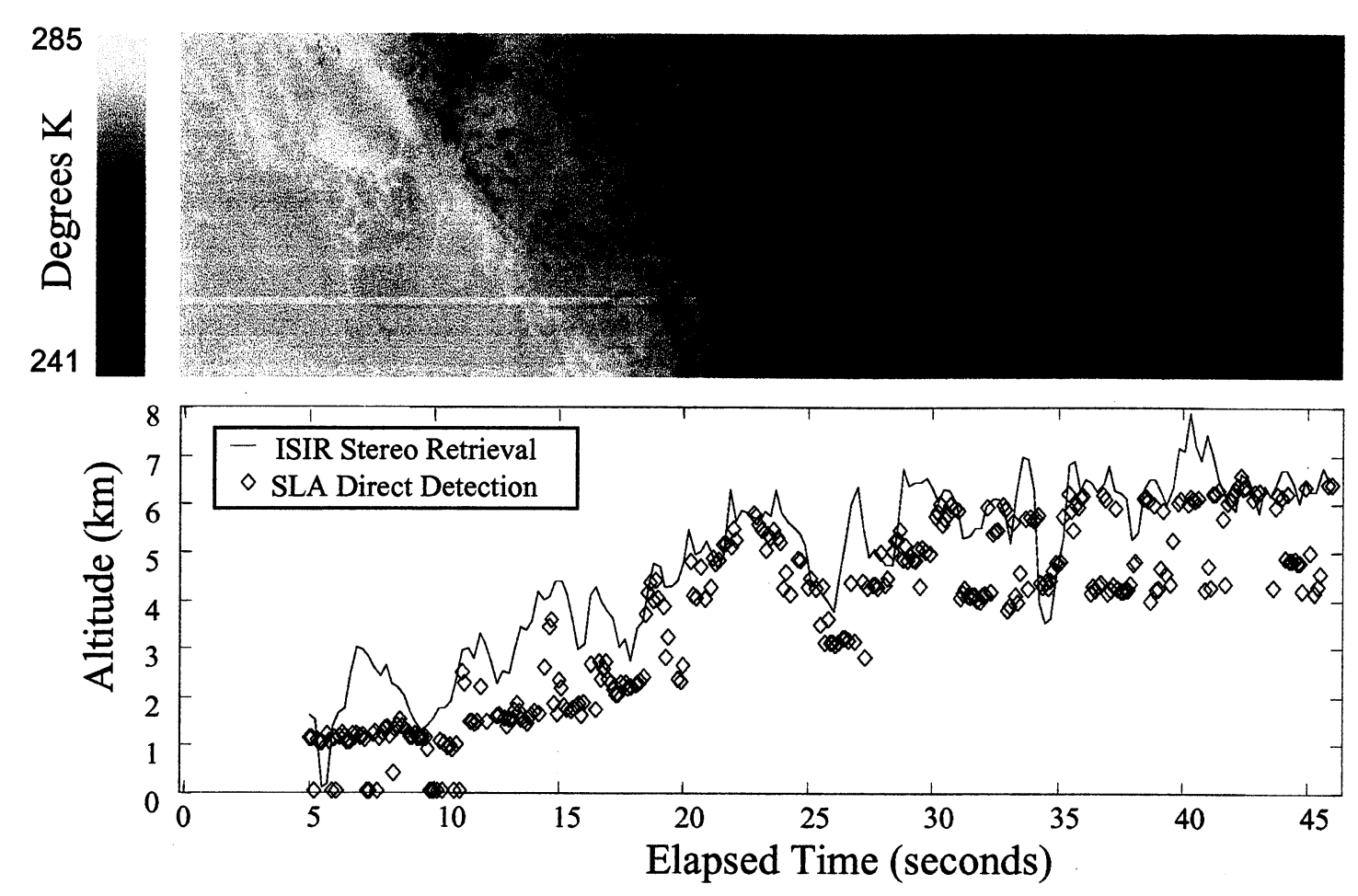

Figure 5. Prior to launch the ISIR instrument was aligned with its FOV centered on the groundtrack of the Shuttle Laser Altimeter to facilitate a comparison of the measurements provided by the two instruments. Shown here is a comparison of cloud height estimates obtained stereoscopically and through direct detection for an optically thick cloud scene that reaches as high as $6 \mathrm{~km}$ in altitude. 

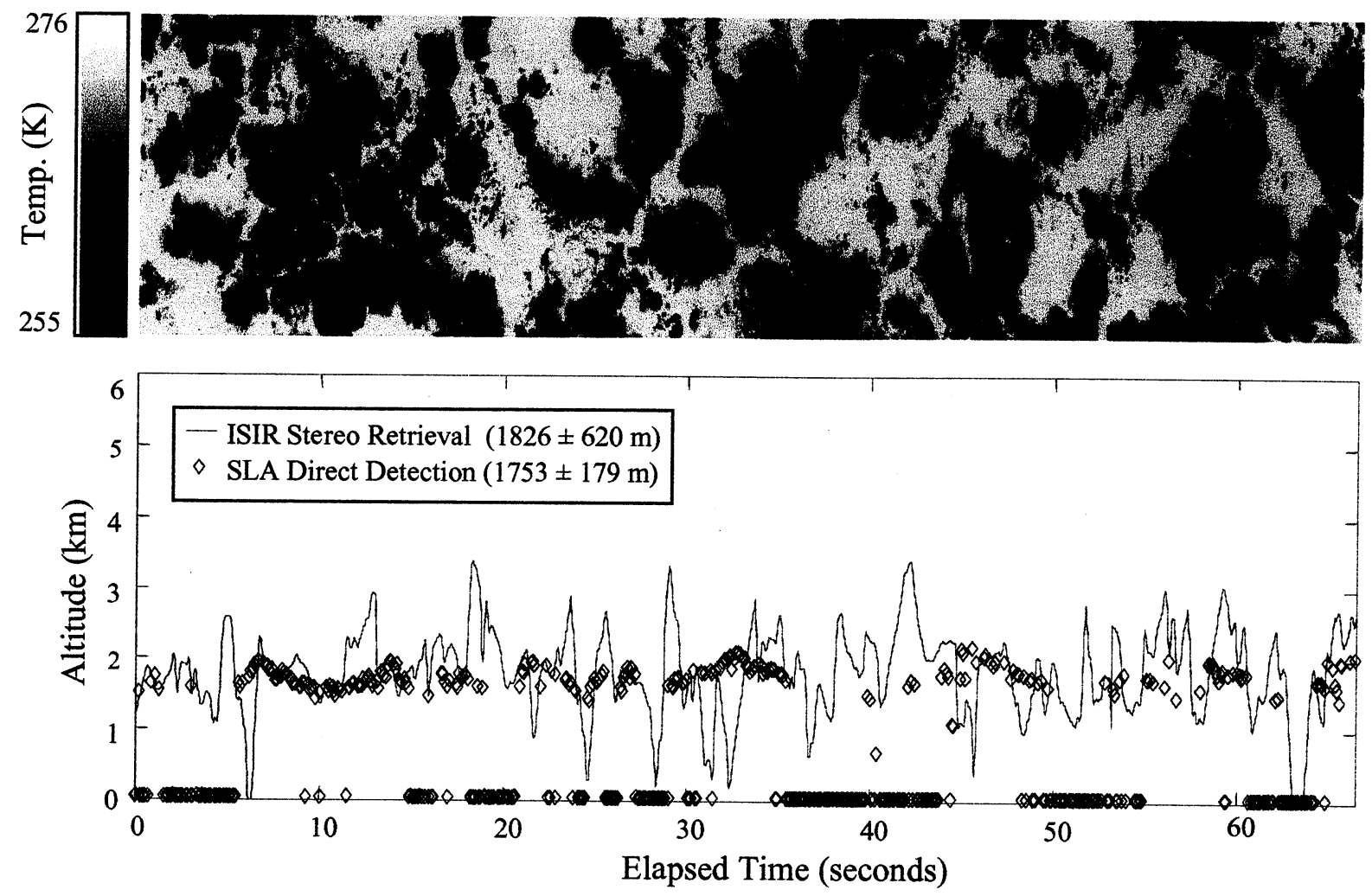

Figure 6. A scene of broken cumulus clouds is used to estimate the vertical resolution of the stereoscopic cloud height measurements by comparing them with direct detection measurements obtained from the Shuttle Laser Altimeter. Inversion of the ISIR stereo imagery results in an average cloud height of $1826 \pm 620 \mathrm{~m}$ whereas the direct detection measurements of the Shuttle Laser Altimeter reveal an average height of $1753 \pm 179 \mathrm{~m}$. 


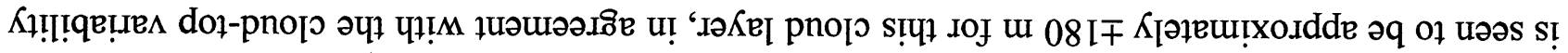

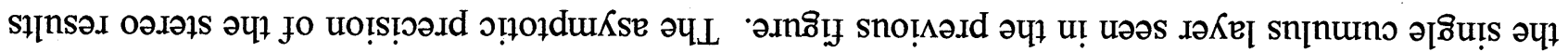

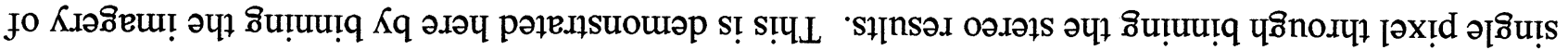

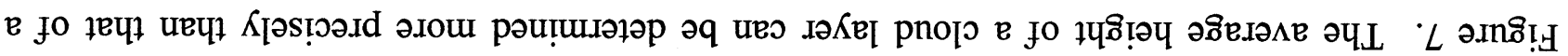

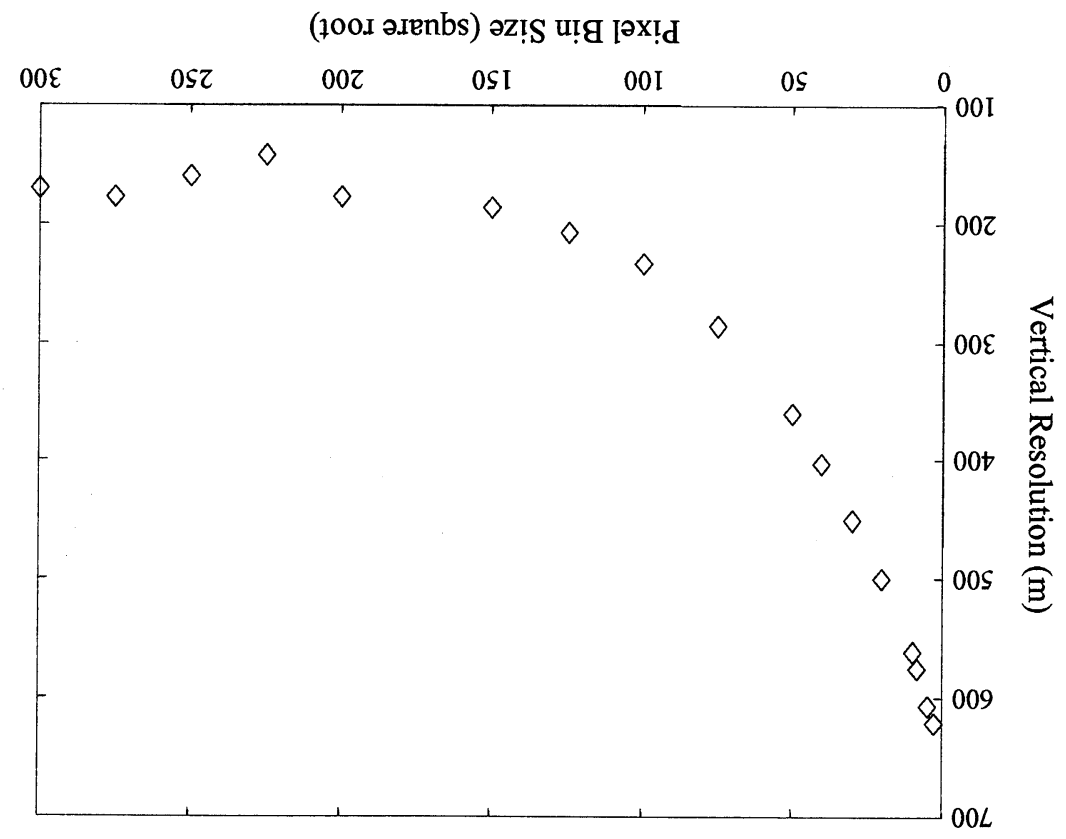




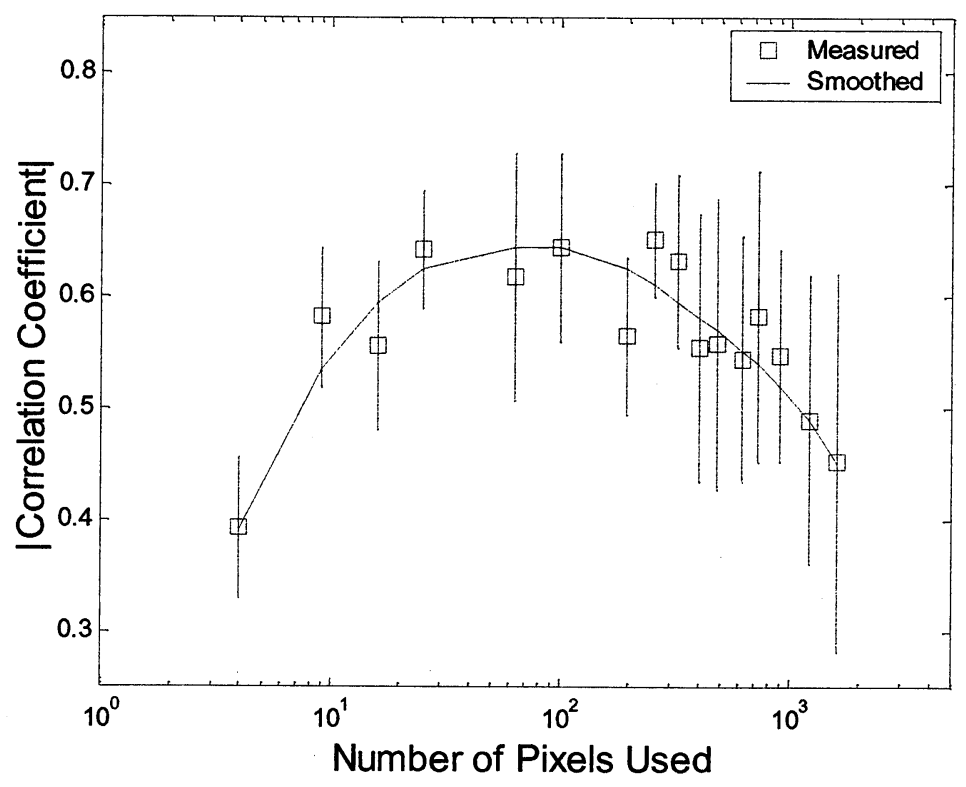

Figure 8 . The correlation between cloud height and brightness temperature provides a means to determine the optimal grid size for use in the registration of the ISIR imagery. Using the imagery of Figure 4 it is seen that an approximate 10x10 -pixel grid provides the best correlation between the brightness temperature and stereoscopically derived cloud height. These results are used to weight the average of stereo height estimates obtained using different grid resolutions. 